\title{
Utilization of laser interstitial thermotherapy guided by real-time thermal MRI as an alternative to separation surgery in the management of spinal metastasis
}

\author{
Claudio E. Tatsui, MD, ${ }^{1}$ R. Jason Stafford, PhD, ${ }^{2}$ Jing Li, MD, ${ }^{3}$ Jonathan N. Sellin, MD, ${ }^{1}$ \\ Behrang Amini, MD, PhD, ${ }^{4}$ Ganesh Rao, MD, ${ }^{1}$ Dima Suki, PhD, ${ }^{1}$ Amol J. Ghia, MD, ${ }^{3}$ \\ Paul Brown, MD, ${ }^{3}$ Sun-Ho Lee, MD, ${ }^{5}$ Charles E. Cowles, MD, MBA, ${ }^{6}$ Jeffrey S. Weinberg, MD, ${ }^{1}$ and \\ Laurence D. Rhines, MD'
}

\begin{abstract}
Departments of ${ }^{1}$ Neurosurgery, ${ }^{2}$ maging Physics, ${ }^{3}$ Radiation Oncology, ${ }^{4}$ Diagnostic Radiology, and ${ }^{6}$ Anesthesiology, The University of Texas MD Anderson Cancer Center, Houston, Texas; and ${ }^{5}$ Department of Neurosurgery, Samsung Medical Center, Sungkyunkwan University School of Medicine, Seoul, South Korea
\end{abstract}

\begin{abstract}
OBJECT High-grade malignant spinal cord compression is commonly managed with a combination of surgery aimed at removing the epidural tumor, followed by spinal stereotactic radiosurgery (SSRS) aimed at local tumor control. The authors here introduce the use of spinal laser interstitial thermotherapy (SLITT) as an alternative to surgery prior to SSRS.

METHODS Patients with a high degree of epidural malignant compression due to radioresistant tumors were selected for study. Visual analog scale (VAS) scores for pain and quality of life were obtained before and within 30 and 60 days after treatment. A laser probe was percutaneously placed in the epidural space. Real-time thermal MRI was used to monitor tissue damage in the region of interest. All patients received postoperative SSRS. The maximum thickness of the epidural tumor was measured, and the degree of epidural spinal cord compression (ESCC) was scored in pre- and postprocedure MRI.

RESULTS In the 11 patients eligible for study, the mean VAS score for pain decreased from 6.18 in the preoperative period to 4.27 within 30 days and 2.8 within 60 days after the procedure. A similar VAS interrogating the percentage of quality of life demonstrated improvement from $60 \%$ preoperatively to $70 \%$ within both 30 and 60 days after treatment. Imaging follow-up 2 months after the procedure demonstrated a significant reduction in the mean thickness of the epidural tumor from $8.82 \mathrm{~mm}(95 \% \mathrm{Cl} 7.38-10.25)$ before treatment to $6.36 \mathrm{~mm}(95 \% \mathrm{Cl} 4.65-8.07)$ after SLITT and SSRS $(p=$ $0.0001)$. The median preoperative ESCC Grade 2 was scored as 4, which was significantly higher than the score of 2 for Grade $1 b(p=0.04)$ on imaging follow-up 2 months after the procedure.
\end{abstract}

CONCLUSIONS The authors present the first report on an innovative minimally invasive alternative to surgery in the management of spinal metastasis. In their early experience, SLITT has provided local control with low morbidity and improvement in both pain and the quality of life of patients.

http://thejns.org/doi/abs/10.3171/2015.2.SPINE141185

KEY WORDS laser interstitial thermotherapy; spinal metastasis; separation surgery; technique

\footnotetext{
ABBREVIATIONS cEBRT = conventional external beam radiation therapy; ESCC = epidural spinal cord compression; HASTE = half Fourier acquisition single-shot turbo spin-echo; K-wire = Kirschner wire; NOMS = neurologic, oncologic, mechanical, systemic; SINS = Spine Instability Neoplastic Score; SLITT = spinal laser interstitial thermotherapy; $\mathrm{SpO}_{2}=$ blood oxygen saturation; SSRS = spinal stereotactic radiosurgery; VAS = visual analog scale. ACCOMPANYING EDITORIAL See pp 397-399. DOI: 10.3171/2015.3.SPINE15210.

SUBMITTED November 21, 2014. ACCEPTED February 18, 2015.

INCLUDE WHEN CITING Published online July 3, 2015; DOI: 10.3171/2015.2.SPINE141185.

DISCLOSURE The authors report no conflict of interest concerning the materials or methods used in this study or the findings specified in this paper. Dr. Rhines is a consultant for Stryker and Globus. Grant funding was provided by the RGK Foundation.
} 
$\mathrm{O}$ VER the last 40 years, significant progress has been made in the management of metastatic disease causing spinal cord compression. In the 1980s, laminectomy for posterior decompression plus radiotherapy, compared with radiotherapy alone, was shown to worsen pain and neurological function. ${ }^{19,42}$ In this context, conventional external beam radiation therapy (cEBRT), which provided a similar or better outcome with less morbidity, became the standard of care., 49,20,42 Unfortunately, radioresistant histologies demonstrated less than favorable responses ${ }^{22,33}$ with a significant subset of patients deriving little palliative benefit or tumor control from this modality alone..$^{24,35}$ In subsequent years, a better understanding of spinal biomechanics and the development of internal spinal fixation revitalized the role of surgery in the management of spinal metastasis, resulting in improved functional outcomes and local control. A randomized controlled trial ${ }^{32}$ demonstrated the superiority of circumferential decompression and stabilization surgery followed by cEBRT in the maintenance and recovery of ambulation, preservation of continence, pain control, and maintenance or restoration of functional performance, compared with cEBRT alone.

A better understanding of radiobiology and technological advancements in image guidance over the last decade has allowed the development of spinal stereotactic radiosurgery (SSRS), in which radiation is contoured to cover a specific target volume with a steep falloff in radiation delivered to surrounding tissues, limiting the toxicity to organs in proximity to the tumor. ${ }^{18,39}$ The delivery of high doses of radiation in single-dose ${ }^{40}$ or hypofractionated regimens has been shown to overcome the radioresistance of certain histologies to fractionated cEBRT. ${ }^{16-18,40}$ Stereotactic body radiation therapy has become an effective tool in the management of spinal metastasis. ${ }^{30,37}$ But despite the highly contoured nature of radiation dose delivery, the predicted falloff of radiation must remain within the constraints of spinal cord tolerance. In the case of epidural disease displacing the spinal cord, the radiation dose must be decreased in the region adjacent to the spinal cord, which could result in a failure to attain tumoricidal radiation dosing in the epidural compartment and lead to treatment failure. ${ }^{9}$ The judicious use of cEBRT, surgery, and SSRS optimized to minimize morbidity in the management of malignant spinal cord compression was popularized by Bilsky et al. in their proposed neurologic, oncologic, mechanical, and systemic (NOMS) decision framework. ${ }^{4,22}$ These authors coined the term "separation surgery" to describe an operation intended to stabilize the spine and remove the epidural tumor adjacent to the dura mater, rather than one aimed at gross-total intralesional resection. Once the patient recovers from surgery, SSRS is contoured to cover the surgical field, and all residual tumor receives adequate tumoricidal doses of radiation since the epidural disease likely to be underdosed has been surgically removed.,5,22,28 Laufer et al. documented a 1-year cumulative incidence of local recurrence of only $4.1 \%$ when separation surgery was used in combination with hypofractionated SSRS (24-30 Gy divided into 3 fractions) and $9 \%$ when surgery was followed by highdose single-fraction SSRS (24 Gy). ${ }^{21}$
Percutaneous procedures for tumor ablation are widely used with the intent of local tumor control, as in the treatment of visceral ${ }^{2,41}$ and bone metastasis. ${ }^{36}$ In these cases imaging modalities are used to implant a heating or cooling probe into a tumor. However, the damage created is not monitored and occurs in an assumed but unverified distribution around the tip of the probe. Previously, the utilization of such techniques in the spine was limited to lesions without significant extension into the epidural space-the prospect of creating unmonitored thermal damage adjacent to the spinal cord being a limiting factor. ${ }^{1,29}$ This application has not gained popularity, as the lesions were more suitable for SSRS, which avoids the risk of damage to neural structures.

An ideal minimally invasive surgical approach to treat spinal metastasis should achieve local tumor control, allow for fast recovery, minimize postoperative pain and morbidity, and curtail delays in initiating or interrupting systemic therapies directed at the primary tumor. Here, we report the use of spinal laser interstitial thermotherapy (SLITT) as an alternative to separation surgery. Our method involves, under CT-based image guidance, placement of a laser probe in the epidural tumor at an approximate distance of $6 \mathrm{~mm}$ from the dural edge. The heating process is monitored in real time with thermal MRI, and once the temperature reaches a critical level at the dural edge, the system deactivates, protecting the spinal cord from thermal damage. This procedure, as with separation surgery, is followed by SSRS in standard doses to cover the gross tumor volume, as if no thermal ablation were performed. If spinal instability is suspected, one can perform percutaneous placement of spinal instrumentation at the same time and then cement augmentation in a second stage. Herein, we report our initial findings on this treatment paradigm, in which most patients had a short hospital stay, pain scores improved rapidly, interruptions in systemic treatment were avoided, and short-term local control was achieved.

\section{Methods}

\section{Patient Population and Evaluation}

This single-institution retrospective analysis was reviewed and approved by the MD Anderson Cancer Center institutional review board. All patients had documented spinal metastasis from histologies considered to have an unfavorable response to cEBRT. Epidural extension of the tumor was scored based on preoperative axial T2weighted or postcontrast MRI sequences, according to the method described by Bilsky et al. ${ }^{6}$ Spinal stability was assessed using the Spine Instability Neoplastic Score (SINS) described by Fisher et al. ${ }^{12}$ Motor function was evaluated according to the Frankel classification. ${ }^{14}$ All cases were presented in a multidisciplinary tumor board conference with neurosurgery, radiation oncology, and radiology personnel, during which everyone agreed that separation of the tumor from the dura prior to SSRS was required to reduce the risk of epidural failure. Patients with an intact neurological condition who were considered to have a high surgical risk given their medical comorbidities were included in the study, as were those with advanced sys- 
temic cancer in whom the magnitude of surgical intervention was believed to be too aggressive and would be poorly tolerated by the patient. Patients with acute neurological deficits and/or a circumferential epidural tumor involving more than 1 vertebral level were excluded. All patients were evaluated using a physical exam and the visual ana$\log$ scale (VAS) for pain before treatment, within 30 and 60 days after treatment, and at all subsequent follow-up visits (in general, every 3 months). A VAS (a 100-mm line with $0 \%$ on the bottom and $100 \%$ on the top) rating the current quality of life, similar to the health state scale component of the EQ-5D, ${ }^{11}$ was applied in the same time intervals. Informed consent was obtained in all patients according to institutional policies. All patients underwent postoperative MRI with contrast at 8 weeks postprocedure. The time between SLITT and the start of SSRS, hospital stay, postablation complications, and subjective patient satisfaction were recorded.

\section{Preoperative Evaluation and Classification of the Status of Systemic Disease}

All patients were clinically monitored, and systemic radiographic evaluations were performed every 3-4 months to restage and evaluate treatment effectiveness or the need for a change in therapy. We reviewed all imaging studies, including bone scans; CT scans of the chest, abdomen, and pelvis; and MR and CT images of the spine. Extraspinal disease was classified as 1) "concurrent" when metastatic disease was identified within 30 days of the diagnosis of the spinal tumor, 2) "progressing" when there was enlargement or an increase in the number of lesions on 2 consecutive imaging studies prior to laser ablation, and 3) "stable" when no enlargement or increase in the number of lesions was observed on 2 consecutive imaging studies prior to the procedure.

\section{Stereotactic Placement of the Laser Probe in the Epidural Space}

This part of the procedure is performed in the preparation room of our intraoperative MRI suite (BrainLab Inc.), which has laminar air flow and positive air pressure and fulfills the institutional requirements for the performance of percutaneous surgical procedures. After general endotracheal anesthesia is induced, standard antibiotic prophylaxis and a dose of $10 \mathrm{mg}$ of dexamethasone are given and maintained for 12 hours. The patient is positioned prone over gel rolls placed in parallel along the patient body axis with the arms tucked to the side. We use the intraoperative MRI transfer table, which is radiolucent. We place the patient over gel rolls to position the spine at a higher level than the arms, allowing clear lateral fluoroscopic images from T-3 to the sacrum without interference from the upper extremity skeleton. A C-arm (Siemens) is connected to the navigation system (BrainLab Inc.). The spinous process immediately above the vertebral body of interest is identified, and under sterile conditions a small midline incision is made to expose the tip of the selected spinous process. A spinous process clamp is securely attached, and a registration array is positioned away from the entry point selected for insertion of the laser probe. A preoperative CT scan of the spine, obtained with a standard navigation protocol, is matched to anteroposterior and lateral fluoroscopic images of the vertebra attached to the registration array, allowing the navigation system to calculate its parameters for automatic registration. Since we do not have exposure of the deep bony structures, we check the accuracy of registration on the surface of the exposed spinous process under the clamp. Once acceptable accuracy is achieved, we set a posterior lateral transpedicular trajectory to reach a target ideally located at a distance $5 \mathrm{~mm}$ from the ventral dural border in the midline of the posterior vertebral body. We use a Jamshidi needle (Codman-Synthes) registered to the stereotactic navigation system, which allows real-time image guidance. The needle is advanced through the soft tissues until it reaches the lamina of the vertebral body affected by tumor. If the sagittal projection of the navigation is compatible with the tactile sensation of needle touching bone, the needle is moved to the lateral and medial projection of the pedicle wall, and its location is confirmed with anteroposterior fluoroscopic images. Next, the Jamshidi needle is advanced into the pedicle until it reaches the medial wall. At this stage, the needle should be entering the epidural space, confirmation and comparison with anteroposterior fluoroscopic images are performed (Fig. 1), and, if accuracy is satisfactory, the needle is advanced to the predefined target. We then remove the central trocar, place a Kirschner-wire (K-wire) through the Jamshidi needle, which is subsequently removed, and use the K-wire as a guide for placing a nonferromagnetic plastic access cannula.
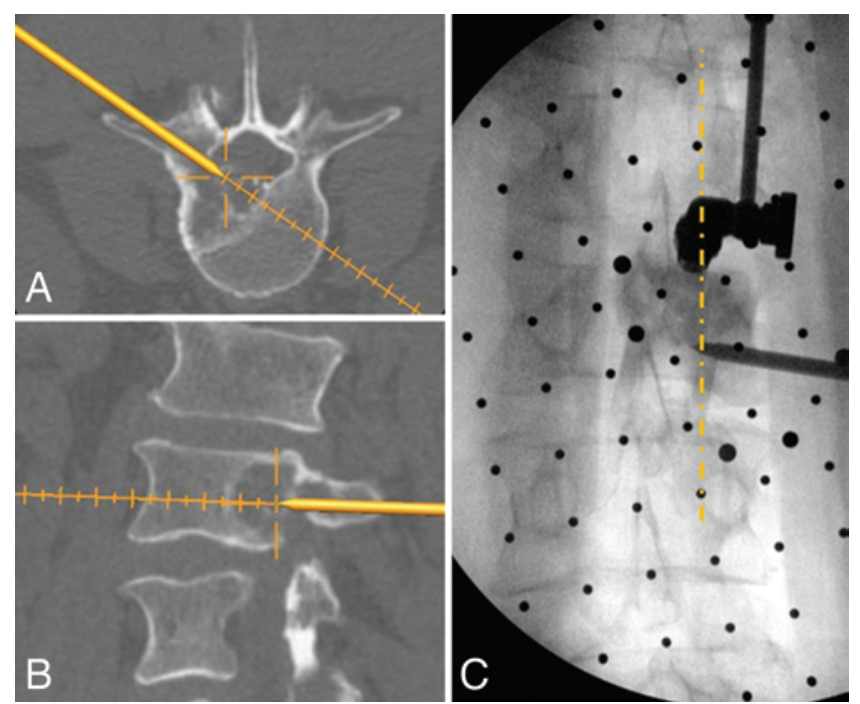

B

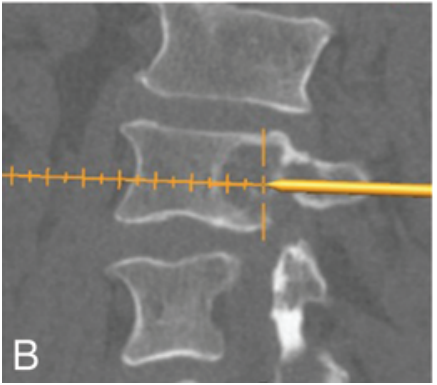

C

FIG. 1. Image guidance technique for percutaneous placement of the laser probe. Axial (A) and sagittal (B) CT reconstructions used for navigation of the Jamshidi needle. Accuracy in the sagittal plane is confirmed when the tactile sensation of needle touching bone corresponds to perfect contact of virtual needle with the cortical surface of the lamina. The needle is advanced until the lateral and medial wall of the pedicle is reached. Fluoroscopic image $(\mathbf{C})$, anteroposterior projection, obtained during needle advancement and compared with the navigation image to ensure accuracy. We use the medial wall of the pedicle inferior to the tumor to draw a reference line (dotted line) for the level of interest. In this example, the navigation image is compatible with the current location of the needle in the inferior portion of the pedicle just passing the medial wall into the vertebral body. Figure is available in color online only. 
In our experience, a distance of $5 \mathrm{~mm}$ on each side of the laser fiber is covered by temperatures associated with tumor cell death. If more coverage is needed, more access cannulas are placed in tandem by using a similar technique to cover the craniocaudal epidural tumor extension. Lastly, the spinous process clamp is removed, an MRIcompatible titanium needle is inserted into the access cannula and docked in residual bone of the vertebral body, and a sterile plastic bag is used to cover the needles.

The thermal images require the use of surface coils placed over the area of interest. We use 2 standard Siemens body matrix coils placed on each side of the patient's back, overlapping in the center and held by straps. The body matrix opening of $10 \times 10 \mathrm{~cm}$ is positioned so there is easy access to the plastic bag covering the sterile needles. The patient is placed in the MRI magnet, and a fast T2-weighted half Fourier acquisition single-shot turbo spin-echo (HASTE) sequence is used to localize the artifacts of the titanium needles, which allows final confirmation of their trajectory and definition of the exact anatomical plane that will be used for the anatomical and thermal sequences (Fig. 2). The exact coordinates of the first needle are stored, the MRI table is backed out of the magnet, the plastic bag is opened, and the first titanium needle is replaced with the laser fiber until it reaches the end of the access cannula, which is pulled back over the fiber for $4 \mathrm{~cm}$ to uncover the tip of the laser probe inside the tumor. During this procedure, blood loss is generally minimal, even in highly vascular lesions such as renal cell carcinoma.

\section{Real-Time Monitoring of Heat by Thermal MRI and Thermal Ablation}

The laser system (Visualase Inc.) is composed of a computer workstation; a 30-W, 980-nm diode laser generator; a cooling pump; and a laser applicator set composed of a 400-nm-core silica fiberoptic with a cylindrical diffusing tip housed within a 1.65 -mm-diameter, saline-cooled polycarbonate catheter.

We use the imaging process described by Ahrar and Stafford. ${ }^{1}$ In brief, heating is monitored by phase-differ- ence imaging with gradient-echo acquisition. The temperature sensitivity of the proton resonance frequency is used to convert changes in phase to estimated changes in tissue temperature on a pixel-by-pixel basis. We use a TR of 38 msec and a flip angle of $20^{\circ}$. The acquisition matrix is 256 $\times 128$ over fields of view of $24-32 \mathrm{~cm}$, typically with a single 3-mm slice acquired every 5-6 seconds, generating a magnitude and a phase image, which are read in real time by the treatment workstation. Extracted information is calculated based on an Arrhenius model and depicted in a color-coded thermal and damage model, which is overlaid with anatomical coplanar T2 images. The software allows monitoring of the temperature in individual pixels. We usually select the pixels in the interface between the epidural tumor and the dura mater (Fig. 3) and set an upper limit of $50^{\circ} \mathrm{C}$; if this temperature is reached, the system automatically deactivates, avoiding thermal damage to the spinal cord and nerve roots.

We observed that any motion significantly deteriorates the thermal map and thus the reliability of the method. To overcome this problem, mechanical ventilation is held during acquisition of the parameters and during thermal damage. We preoxygenate the patient to a blood oxygen saturation $\left(\mathrm{SpO}_{2}\right)$ of $100 \%$ before holding mechanical ventilation. We use capnography curves to track ventilatory movements. Once the curve shows apnea, we activate the laser fiber at $65 \%-70 \%$ of the $30 \mathrm{~W}$ of potency with a maximum pause in ventilation of 100 seconds. At this point, ventilation is resumed and the laser fiber is withdrawn $5 \mathrm{~mm}$. This cycle is repeated 3-4 times to ensure adequate thermal coverage before switching the fiber to the next access cannula. The procedure is interrupted and ventilation is immediately resumed if $\mathrm{SpO}_{2}$ falls below 94\% or if the temperature reaches the predefined temperature threshold next to the spinal cord (automatic deactivation). No anesthesia-related morbidity was observed.

\section{Immediate Estimation of Thermal Damage}

Upon completion of thermal ablation, the patient is returned to the magnet, and T1-weighted (pre- and postcontrast) sequences of the treated area are obtained in respira-
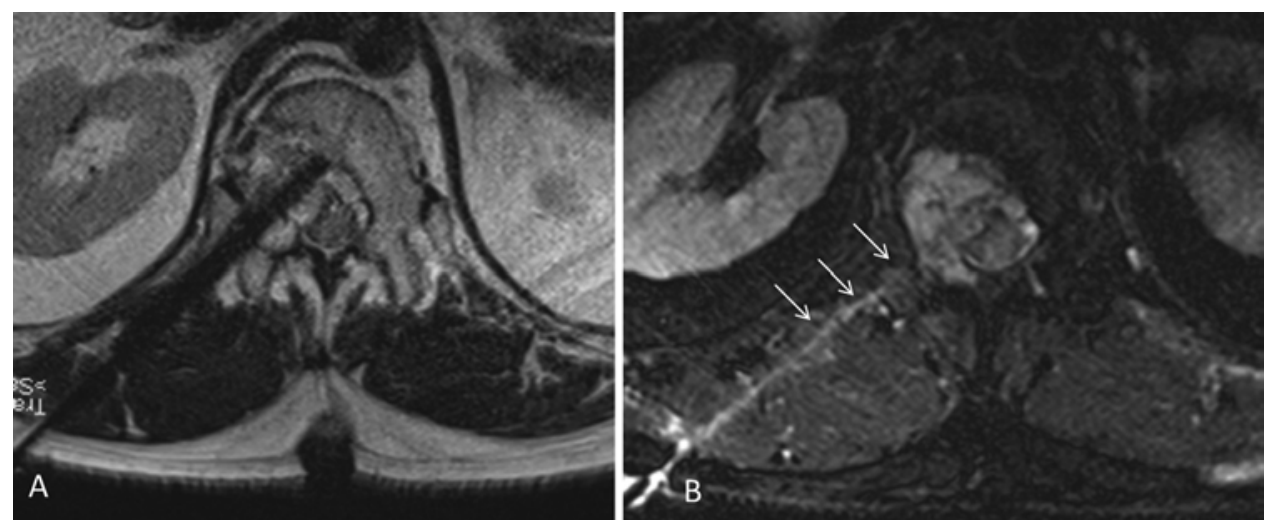

FIG. 2. Intraoperative localization of the laser fiber. Axial T2-weighted HASTE image (A) demonstrating the titanium needle artifact in the epidural tumor. The coordinates are stored, and the titanium insert is removed and replaced by the laser fiber. Axial T1weighted MR image with fat suppression (B) used to localize the laser fiber (arrows) based on coordinates obtained from the same location in the image in panel $A$. 

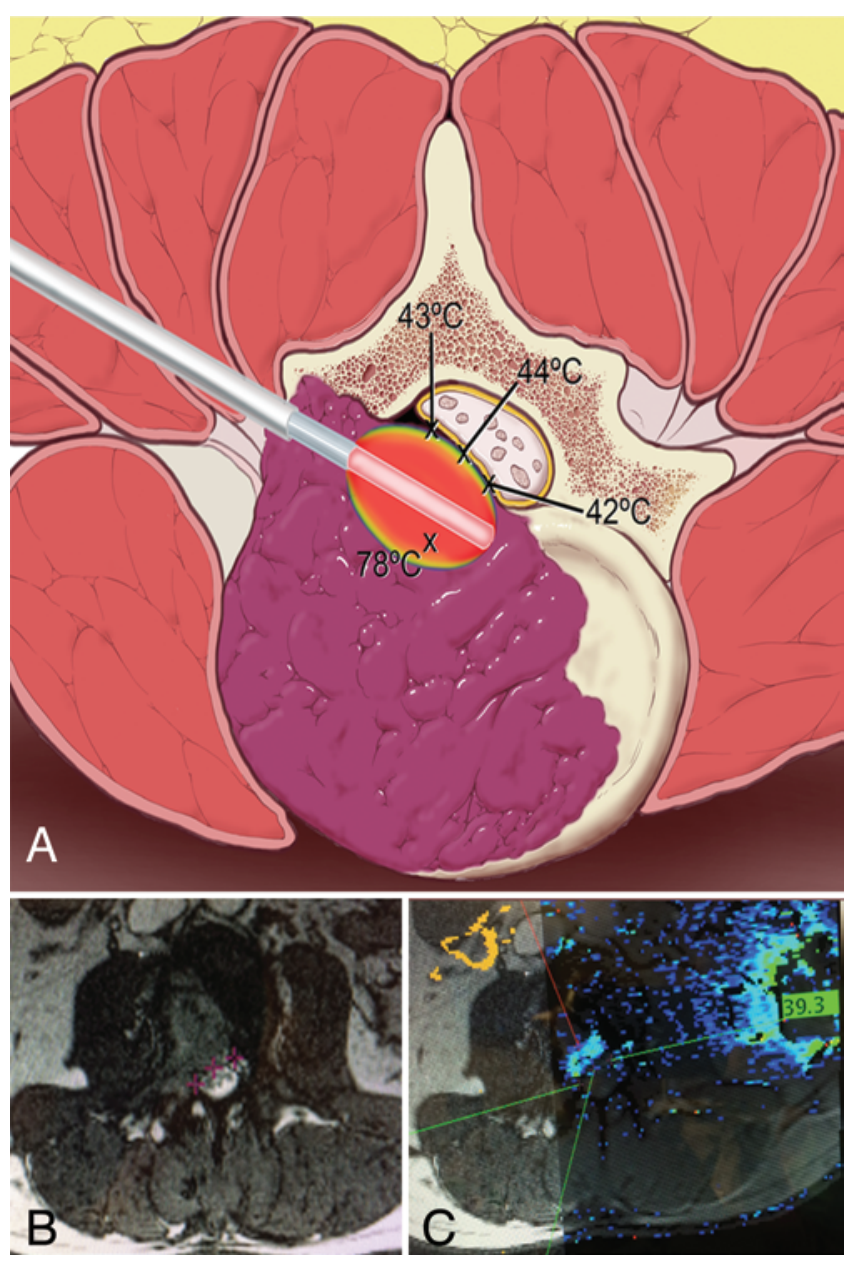

FIG. 3. Intraoperative temperature monitoring. A: Illustration demonstrating final placement of the laser probe in the epidural tumor. We monitor the temperature in 3 points at the junction between the tumor and the dura, with a safety limit of $50^{\circ} \mathrm{C}$. The temperature near the laser fiber is monitored, and a limit of $90^{\circ} \mathrm{C}$ is set to avoid vaporization of the tissue, which prevents adequate spread of the heat. (2014 The University of Texas M.D. Anderson Cancer Center. B: Axial T2-weighted image used to select points near the dura, which will be monitored to protect the neural elements. C: Intraoperative footage of the thermal map overlaid to the corresponding anatomical T2 image, demonstrating real-time monitoring of the spread of heat and temperature at the selected points in the epidural space.

tory arrest. We fuse the images and subtract the nonenhancing tissue to estimate the area or volume of tumor that has been immediately ablated by the treatment (Fig. 4A). This subtraction image emphasizes the degree of immediate necrosis to estimate the total amount of tumor ablated (Fig. 4B). A 5-mm radius of thermal damage is created per fiber.

\section{Delivery of Spinal Stereotactic Radiation}

Preoperative CT and MR images of the spine are used to outline the gross and clinical target volumes. Overall, the fractionation regimen is prescribed based on prior irradiation history, tumor radiosensitivity, tumor volume, and number of vertebral bodies involved. All patients received either a single fraction (24 Gy) or hypofractionation (24-
27 Gy in 3 doses), and most patients were treated within 3 days of the ablation. The treatment plan was calculated to limit the spinal cord dose to $12 \mathrm{~Gy}$ and to maximally cover the gross target volume, as if no ablation had been performed.

\section{Evaluation of Degree of Epidural Tumor Compression}

We obtained MR images with and without contrast preoperatively and every 8 weeks after the complete treatment (ablation and SSRS). To quantify epidural compression, we outlined the contours of the spinal canal and measured the thickness of the epidural tumor on the axial images with the highest degree of spinal canal narrowing. All patients had the degree of epidural spinal cord compression (ESCC) scored using criteria established by Bilsky et al:: ${ }^{6}$ Grade 0, tumor completely contained inside the vertebral body; Grade 1a, tumor extending to the epidural space without displacement of the dura; Grade 1b, tumor compressing the dura without touching the spinal cord; Grade 1c, tumor compressing the dura and touching the spinal cord without displacement; Grade 2, tumor compressing and displacing the spinal cord, but with a column of CSF visible posterior to the cord; and finally Grade 3, epidural compression displacing the spinal cord with complete obliteration of the CSF space. For tumors caudal to the conus medullaris, we adapted the scoring system by adding a virtual spinal cord (with the same area measurement at T-10) in the center of the spinal canal. The epidural tumor was classified based on the mass effect in relation to this virtual spinal cord.

\section{Statistical Analysis}

Statistical analysis was performed using a paired Student t-test for the mean thickness of the epidural space and a Wilcoxon signed-rank test for the median change in the Bilsky classification and changes in the VAS scores for pain and quality of life. A p value $<0.05$ was considered significant.

\section{Results}

\section{Patient Population}

We treated 11 patients (9 males and 2 females) with this technique. The median age was 56 years (range $33-78$ years). Six patients had renal cell carcinoma, 2 had pheochromocytoma, and 1 patient each had melanoma, synovial sarcoma, and hepatocellular carcinoma. The thoracic spine was the most commonly mobile spine segment ( 7 cases), followed by the lumbar spine (3 cases) and cervical spine (1 case; Table 1).

\section{Tumor Location in Relation to Spinal Cord and Position of the Probe}

The location of the tumor influenced the direction of approach in placing the laser probe. Tumors located in the pedicle and posterolateral vertebral body were more easily accessed than tumors located centrally at the midline. Lesions located in the lamina were accessed through a contralateral translaminar approach. The presence of hardware (titanium or platinum) can influence the quality 

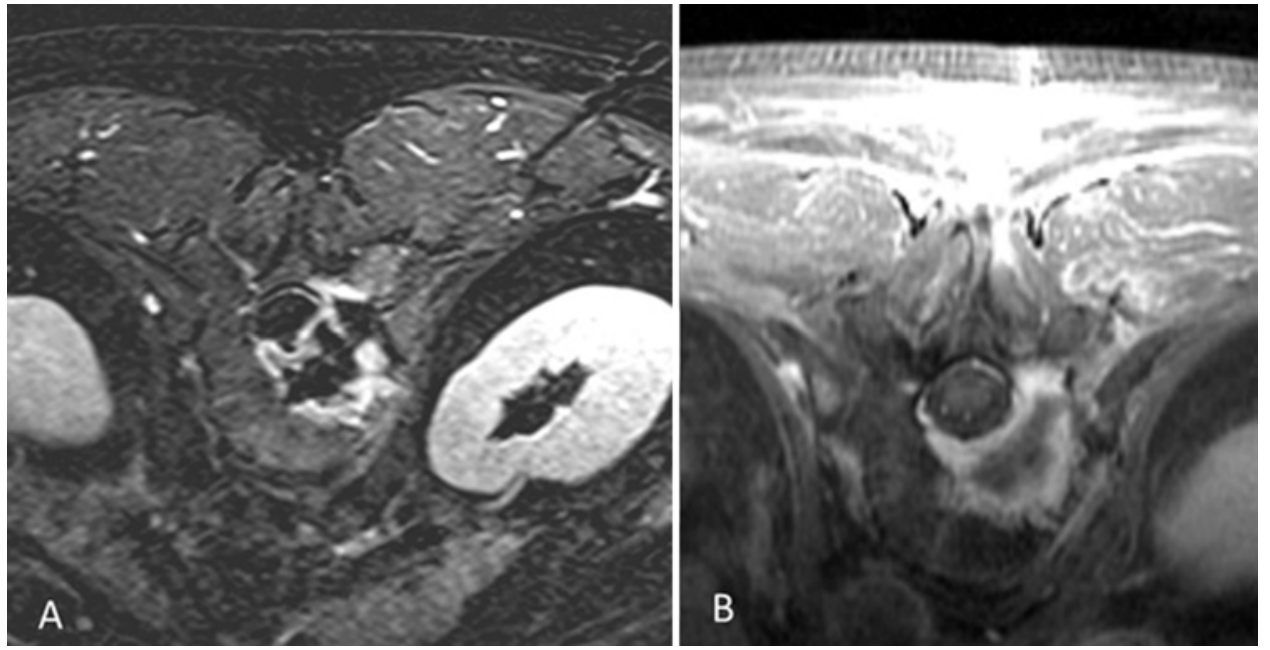

FIG. 4. Estimation of immediate thermal damage and correlation with standard image follow-up. A: Immediate axial T1-weighted postcontrast subtraction image (precontrast image subtracted from the postcontrast image). Subtraction emphasizes the enhancing areas and shows immediate postprocedure coagulative necrosis to better advantage. Note that there is mild ventral epidural mass effect. B: Axial T1-weighted image with contrast at the same level as the image in panel A obtained 2 months after the ablation, demonstrating resolution of the epidural mass effect.

of the temperature mapping since the gradient echo sequence used to acquire the thermal imaging is much more sensitive to metal than fast spin echo T1- or T2-weighted sequences. One patient, who presented with a recurrent synovial sarcoma at C-2, had undergone 2 prior attempts at en bloc resection that were supplemented with instrumentation as well as an ipsilateral vertebral artery embolization, leaving titanium screws contralateral to our approach (C1-3) and platinum coils at the level of C-1 and C-5. In this case, however, we were able to obtain a window without artifact at the exact level of tumor recurrence, which allowed for good thermal mapping. In our experience, tumors in the thoracic spine and thoracolumbar junction are easier to access than those in the lower lumbar spine, which usually require an extreme lateral approach to reach the epidural space. In all cases, however, a transpedicular route was used, the lone exception being the aforemen- tioned patient who underwent a C-2 ablation, in whom a direct intratumoral puncture was used.

\section{Status of Systemic Disease}

Seven patients had progressive systemic disease and were in the process of changing chemotherapy regimens because of systemic failure. The most common site of disease progression was the lungs ( 5 patients), followed by the liver ( 2 patients) and extraspinal bone (2 patients). In all cases, the performance of open spinal surgery would have delayed the initiation of systemic treatment.

Three patients were classified as having a concurrent diagnosis of the primary tumor ( 2 renal cell carcinomas and 1 pheochromocytoma) and spinal and extraspinal metastasis. They all underwent open abdominal surgery to remove the primary tumor less than 2 weeks after the spinal ablation.

TABLE 1. Demographic summary for 11 patients with epidural compression

\begin{tabular}{|c|c|c|c|c|c|c|c|c|c|}
\hline $\begin{array}{l}\text { Case } \\
\text { No. }\end{array}$ & $\begin{array}{l}\text { Age (yrs)/ } \\
\text { Sex }\end{array}$ & Primary Tumor & Level & $\begin{array}{l}\text { Preop KPS } \\
\text { Score (\%) }\end{array}$ & SINS & $\begin{array}{c}\text { Hospital } \\
\text { Stay (days) }\end{array}$ & $\begin{array}{l}\text { Time From Op } \\
\text { to SSRS (days) }\end{array}$ & $\begin{array}{c}\text { SSRS Dose } \\
\text { (Gy/Fr) }\end{array}$ & Remark \\
\hline 1 & $54 / F$ & RCC & L-3 & 60 & 8 & 1 & 1 & $24 / 3$ & \\
\hline 2 & $71 / \mathrm{M}$ & Melanoma & $\mathrm{T}-2$ & 50 & 10 & 1 & 26 & $24 / 3$ & Prior cEBRT \\
\hline 3 & $64 / M$ & RCC & T-10 & 90 & 7 & 2 & 4 & $24 / 1$ & \\
\hline 4 & $78 / M$ & $\mathrm{RCC}$ & T12-L1 & 90 & 8 & 2 & 3 & $27 / 3$ & \\
\hline 5 & $33 / F$ & Synovial SA & $\mathrm{C}-2$ & 90 & NA & 1 & 45 & $24 / 3$ & Prior fusion \& cEBRT \\
\hline 6 & $46 / M$ & $\mathrm{RCC}$ & T-12 & 60 & 12 & 16 & 3 & $24 / 1$ & Followed by nephrectomy \\
\hline 7 & $57 / M$ & RCC & $\mathrm{T}-4$ & 90 & 5 & 4 & 3 & $24 / 1$ & \\
\hline 8 & $56 / M$ & RCC & L-4 & 50 & 9 & 7 & 3 & $24 / 1$ & \\
\hline 9 & $58 / \mathrm{M}$ & Pheocromo & L-3 & 90 & 5 & 1 & 3 & $24 / 1$ & \\
\hline 10 & $52 / \mathrm{M}$ & HepatoCA & T-12 & 60 & 10 & 3 & 2 & $24 / 1$ & \\
\hline 11 & $39 / M$ & Pheocromo & T-11/T-12 & 80 & 7 & 8 & 60 & $27 / 3$ & $\begin{array}{l}\text { Followed by adrenalectomy, prior } \\
\text { cEBRT }\end{array}$ \\
\hline
\end{tabular}

$\mathrm{Fr}=$ fraction; HepatoCA = hepatocellular carcinoma; KPS = Karnofsky Performance Scale; NA = not available; Pheochromo = pheochromocytoma; RCC = renal cell carcinoma; Synovial $\mathrm{SA}=$ synovial sarcoma. 


\section{Grading ESCC and Spinal Instability}

All patients had an epidural tumor compressing the spinal cord or cauda equina. The dura mater was pushed by the tumor touching the spinal cord in 3 patients (ESCC Grade 1c). Four patients had the tumor displacing the spinal cord with a column of CSF in the posterior aspect of the spinal cord (ESCC Grade 2), whereas 4 patients had no CSF visible around the spinal cord (ESCC Grade 3).

The SINSs were recorded, 3 patients were considered stable (SINS 0-6), and 8 patients were scored as potentially unstable (SINS 7-12). One patient was considered to have a high risk of instability (SINS 12) and underwent percutaneous placement of pedicle screws at the time of laser ablation. One patient had en bloc resection and cervical spinal stabilization in a prior operation outside our institution and was considered stable by virtue of the prior instrumentation. Finally, 1 patient developed significant mechanical pain 2 months after the ablation and underwent placement of percutaneous pedicle screws supplemented with vertebroplasty.

\section{Duration of the Procedures}

All patients were admitted on the same day of the procedure. The average length of the procedure was 8 hours. This time included patient anesthesia and positioning, obtaining the fluoroscopic match for navigation, placing the access cannulas into the desired location in the epidural tumor, transferring the patient into the MRI magnet, obtaining the localization for each fiber, obtaining parameters for the thermal map for each fiber, performing an average of 5 cycles of heating per puncture with ventilator pauses, evaluating somatosensory evoked potential monitoring between each cycle, obtaining a final scan with and without contrast to evaluate the ablated tissue, closing the stab wounds, and transferring the patient to a stretcher for extubation. All patients recovered well, without deficits or complaints. Pain was minimal, and all patients had a short hospital stay, unless they had been admitted for concurrent resection of their primary tumor (nephrectomy in Case 6 and adrenalectomy in Case 11).

\section{Estimated Immediate Tumor Ablation}

The ablated tumor can be estimated by the final axial T1-weighted postcontrast subtraction MR images (Fig. 4). The area of nonenhancement on this image corresponds to tumor that was immediately destroyed by the ablation. Given our observations, we estimate that we ablated on average an area equivalent to $36.2 \%$ of the total tumor volume in each patient. Placing the laser probe in the epidural space can create a 10-mm-diameter lesion around the laser probe, and the total area of damage is proportional to the number of fibers (Table 2).

\section{Morbidity and Hospital Stay}

All individuals were admitted to regular hospital beds after the procedure, and the median hospital stay was 2 days (range 1-16 days). The majority of our patients experienced a short hospital stay and were discharged once pain was under control and they were capable of ambulating without assistance. Two patients underwent surgery to address the primary tumor in the same hospital stay, and they remained hospitalized until they recovered from the abdominal surgery (Table 1). All patients had a preoperative Frankel grade of E, and this score remained unchanged in the postoperative period. The patient with cervical synovial sarcoma (Case 5), in whom the laser probe was directly placed in the tumor, experienced tingling in the entire lower extremity and posterior aspect of the right arm, sparing the hands and feet, with decreased perception of temperature that started 72 hours after the procedure. On examination, this patient was otherwise neurologically intact, displaying no changes in motor coordination, proprioception, gait, muscle strength, reflexes, or pain perception. Given this complaint, we performed MRI of the cervical spine, which demonstrated near complete ablation of the tumor, without progression of the epidural disease or an increase in spinal cord compression; however, a faint $\mathrm{T} 2$ hyperintensity in the left lateral aspect of the spinal cord at the level of C-2, which had not been present on the immediate post-ablation image, was observed. We believe this cord signal change represented a delayed effect of the thermal ablation, which caused mild

TABLE 2. Detail of treatment parameters in 11 patients who underwent SLITT

\begin{tabular}{cccccc}
\hline Case No. & $\begin{array}{c}\text { Estimated Tumor } \\
\text { Area }\left(\mathrm{cm}^{2}\right)\end{array}$ & $\begin{array}{c}\text { Estimated Ablated } \\
\text { Area }\left(\mathrm{cm}^{2}\right)\end{array}$ & $\begin{array}{c}\text { Percentage of Tumor } \\
\text { Ablated }\end{array}$ & No. of Fibers & $\begin{array}{c}\text { Average Distance From } \\
\text { Fibers to Dura }(\mathrm{mm})\end{array}$ \\
\hline 1 & 27.48 & 8.02 & 29.1 & 2 & 9.5 \\
\hline 2 & 5.1 & 1.00 & 19.6 & 2 & 5.8 \\
\hline 3 & 6.94 & 1.57 & 22.6 & 3 & 5.2 \\
\hline 4 & 13.95 & 2.85 & 20.4 & 4 & 5.8 \\
\hline 5 & 1.61 & 1.12 & 69.6 & 3 & 5.1 \\
\hline 6 & $\mathrm{NA}$ & $\mathrm{NA}$ & 22.3 & 4 & 5.2 \\
\hline 7 & 8.67 & 1.93 & 23.5 & 3 & 5.0 \\
\hline 8 & 8.21 & 2.06 & 36.2 & 2 & 5.7 \\
\hline 9 & 6.29 & 2.28 & 69.4 & 2 & 5.7 \\
\hline 10 & 10.03 & 6.97 & 52.1 & 2 & 4.8 \\
\hline Median & 4.89 & 2.55 & 36.2 & 2 & 5.7 \\
\hline
\end{tabular}


edema in the spinal cord and partial dysfunction of the lateral spinothalamic tract sparing the anterior spinothalamic tract. We delayed the SSRS for 4 weeks until we confirmed complete resolution the T2 abnormality with new MRI. The patient's symptoms had subsided by the 4-week follow-up.

One patient with renal cell carcinoma (Case 6) previously treated with bevacizumab presented with postoperative dehiscence and infection of the midline incision made to anchor the spinous process clamp used for image guidance. This patient underwent debridement, received intravenous antibiotics, and underwent placement of a wound vacuum, and healing occurred by secondary intention.

\section{Postprocedure Image Control and Treatment Failures}

Follow-up MRI was performed 8 weeks after the procedure in all patients. We noted that all individuals developed an area of central coagulative necrosis, with peripheral contrast enhancement outlining the treated region. We measured the thickness of the epidural tumor at the level of maximal epidural compression before and after treatment in all patients (Table 3 ). We noted a mean reduction of $27.8 \%$ in the median thickness of the epidural tumor on the 8-week follow-up images. The mean thickness of the epidural tumor decreased significantly from $8.82 \mathrm{~mm}(95 \%$ CI $7.38-10.25)$ prior to treatment to 6.36 $\mathrm{mm}$ (95\% CI 4.65-8.07) on the 2-month follow-up images $(\mathrm{p}=0.0001)$. Our current median follow-up is 4.7 months (range 3.4-6.6 months), and no change in the thickness of this epidural enhancement was noted in the patients with image follow-up beyond 8 weeks from the procedure.

The original ESCC score ranged from 0 to 3, but later Grade 1 was subdivided into Grades 1a, 1b, and 1c. To quantify improvement based on the ESCC grade, we converted each of the 5 ESCC grades to a score: Grade 0 was converted to a score of 0 , Grade 1a to a score of 1 , Grade $1 \mathrm{~b}$ to a score of 2 , Grade $1 \mathrm{c}$ to a score of 3 , Grade 2 to a score of 4, and Grade 3 to a score of 5. The median preoperative converted ESCC score of 4 (range 3-5, equivalent to ESCC Grade 2) was significantly higher than the median converted ESCC score of 2 (range 1-5, equivalent to an ESCC Grade 1b) observed on the 2-month follow-up image ( $\mathrm{p}=0.04$, Wilcoxon test; Table 3).

One patient with renal cell carcinoma metastatic to L-3 (Case 8) had a myelographic blockage (ESCC Grade 3) prior to treatment. This individual developed intractable radicular pain despite a modest reduction in the thickness of the epidural tumor on the follow-up image at 4 weeks after ablation and SSRS. He underwent a posterior transpedicular vertebrectomy with polymethylmethacrylate reconstruction of the vertebral body and pedicle screw stabilization, with significant improvement in his pain and performance status. Another patient with hepatocellular carcinoma had an unchanged epidural tumor (stable). All other patients (9 of 11) had a favorable response, with a decrease of at least 1 grade in the ESCC.

\section{Pain and Quality of Life Measures}

The mean preoperative VAS for pain was 6.18 (SD 2.27), which was significantly higher than the score of 4.27 (SD 2.32, $\mathrm{p}=0.035)$ observed within 30 days or the
TABLE 3. Clinical and radiographic follow-up parameters in 11 patients after SLITT

\begin{tabular}{cccccc}
\hline \multirow{2}{*}{$\begin{array}{c}\text { Case } \\
\text { No. }\end{array}$} & \multicolumn{2}{c}{ ESCC Grade } & & Greatest Epidural Tumor Thickness (mm) \\
\cline { 6 - 6 } \cline { 5 - 6 } & Preop & 2 Mos & & Preop & 2 Mos \\
\hline 1 & 2 & 1b & & 9 & 5 \\
\hline 2 & 3 & 3 & 9 & 6 \\
\hline 3 & 1c & 1a & 6 & 2 \\
\hline 4 & 1c & 1b & 8 & 7 \\
\hline 5 & 2 & 1c & 11 & 8 \\
\hline 6 & 2 & 1b & 8 & 4 \\
\hline 7 & 3 & 3 & 8 & 6 \\
\hline 8 & 3 & 3 & 14 & 12 \\
\hline 9 & $1 \mathrm{c}$ & 1b & 7 & 6 \\
\hline 10 & 2 & 3 & 8 & 8 \\
\hline 11 & 3 & 1b & 9 & 6 \\
\hline Median & 2 & 1b & 8 & 6 \\
\hline
\end{tabular}

score of 2.8 (SD 1.88, p = 0.01) obtained within 60 days. The VAS rating the quality of life remained stable without significant changes at $60 \%$ preoperatively and $70 \%$ within both $30(\mathrm{p}=0.09)$ and 60 days after treatment $(\mathrm{p}=0.31$; Fig. 5). Most of the initial postoperative scores were obtained within 7 days from the ablation; therefore, we believe that the improvement observed in our patients-at least in this short interval-represents the effect of ablation. One patient underwent a nephrectomy and 1 had an adrenalectomy 2 weeks after our procedure, and we believe the pain and quality of life scores obtained within 60 days were negatively influenced by these procedures.

\section{Discussion}

The management of metastatic disease to the spine is multimodal and involves a multidisciplinary approach with roles played by oncologists, who deliver chemotherapy, hormonal therapy, and/or immunotherapy in an attempt to achieve systemic control; radiation oncologists, who deliver radiation to the tumor, aiming at local control; and surgeons, who provide spinal stabilization and immediate neural element decompression, aiming at the preservation of neurological function, spinal stability, and symptom relief. ${ }^{22,28}$ Unfortunately, these aims must be balanced against each other, with local control and functional preservation generally taking priority over systemic control in most acute presentations. In cases with a high degree of spinal cord compression, the most common surgical approach for the thoracic and lumbar spine involves a laminectomy with pedicle screw stabilization; $3,13,42$ for the cervical spine, an anterior approach with vertebral column reconstruction..$^{27,31}$ In either case, surgical intervention delays systemic treatment (usually for 3-4 weeks) while patients recover functional status, wounds heal, and postoperative radiation is delivered.

Knowledge of tumor histology is fundamental in the management of spinal metastasis. Even in cases of a suspected primary tumor, histological confirmation is needed for treatment planning. In general, radiation is well toler- 

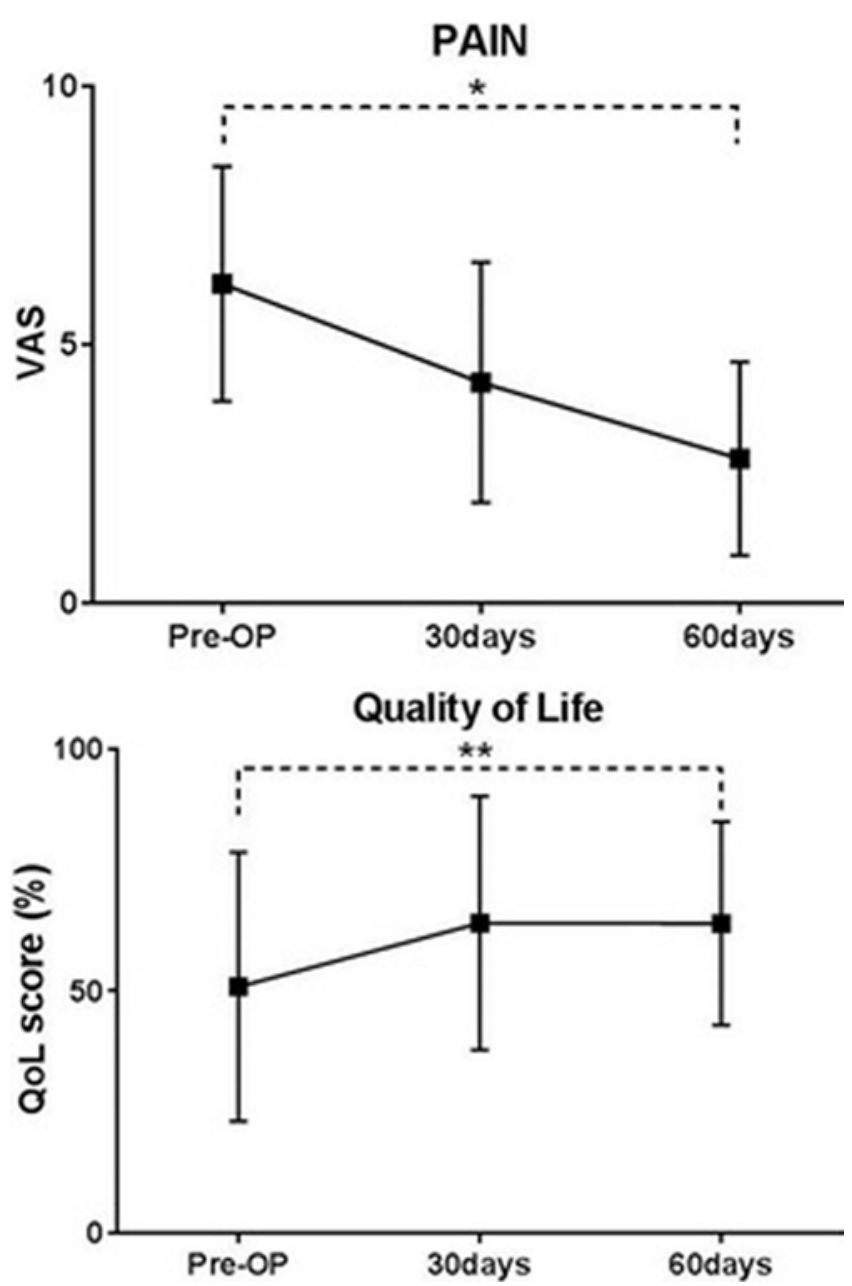

FIG. 5. Visual analog scale for pain and quality of life. Upper: Graph demonstrating the VAS score for pain over time. Lower: Graph demonstrating the VAS score for quality of life over time. ${ }^{*} p<0.05 .{ }^{* *} p>0.05$. Statistical significance was determined using the Wilcoxon signed-ranks test. QoL = quality of life.

ated by patients, and the conventional regimen of $30 \mathrm{~Gy}$ in 10 fractions is commonly used even in cases of terminal disease. ${ }^{25,33,34}$ The notion that conventional radiotherapy confers a palliative benefit comes from studies that compared this modality with lumbar and thoracic laminectomy and used the maintenance and recovery of ambulation as an outcome measure. ${ }^{4,19,20,23,24,42}$ It is well known that some histologies are more sensitive to conventional radiation (breast, prostate, hematological) than others (lung, renal cell carcinoma). ${ }^{19,22,24,34}$ However, recent advancements in technology have allowed for the delivery of high doses of highly conformed radiation capable of overcoming this radioresistance to conventional radiotherapy. Recent series have demonstrated improvement in local control, pain, and the quality of life of patients treated with this modality. ${ }^{15-18,30,37,39,40}$

Bilsky and Smith ${ }^{4}$ have proposed a decision framework (NOMS) based on neurological, oncological, mechanical, and systemic considerations ${ }^{22}$ in which the judicious use of surgery and conventional and stereotactic radiation are combined to provide optimal local control, symptomatic relief, and functional preservation for patients suffering from spinal metastasis. ${ }^{4,5,21,22,28}$ These authors advocate surgery only in cases with a high degree of spinal cord compression in recognition of the fact that tumoricidal doses of radiation can be delivered with the stereotactic method up to but not including a margin of 2-3 mm around the spinal cord. ${ }^{4,5}$ In cases where radioresistant histologies with epidural extension have violated this 2- to 3-mm margin, they advocate a surgical approach in which the goal is spinal stabilization and resection of the epidural tumor, but not gross-total resection (separation surgery). This approach avoids complex reconstruction of the vertebral bodies and decreases surgical time and blood loss, thus facilitating a more rapid recovery from surgery. ${ }^{4,5,22,28}$ Laufer et al. have demonstrated that separation surgery in combination with stereotactic radiation provides 1 -year local control rates of more than $91 \%$ regardless of tumor histology radiosensitivity. ${ }^{21}$

Laser thermal therapy has been used to treat cranial, 7,8 liver, ${ }^{38}$ and bone ${ }^{1}$ lesions. The biggest advantage of this modality is its compatibility with MRI. As heat is generated in tissue, changes in the MR can be translated into a thermal map and overlay of coplanar anatomical images, allowing real-time monitoring of the intensity and spread of heat in the tissue as the laser is fired.$^{26}$ This technology has been used in neurosurgery to treat intracranial metastases, ${ }^{8}$ primary brain tumors, ${ }^{7}$ and epilepsy $y^{10}$ with excellent results and low morbidity. Ahrar and Stafford ${ }^{1}$ have reported the use of laser interstitial thermotherapy (LITT) to treat spinal metastasis, but their study excluded tumors extending to the epidural space. These authors concluded that LITT is a safe and reliable technique, but they recognized the technical challenges in placing a probe inside the tumor, as they used a freehand technique guided by MRI. This application never garnered extensive interest, since SSRS provided better accuracy for and local control of the tumors selected for their study.

We report for the first time the use of LITT in the treatment of tumors located in the epidural space of the spinal canal. As with surgery, we do not advocate LITT as a single treatment modality. All of our patients underwent SSRS after ablation, and it is our intention that the 2 treatments overlap in a synergistic fashion; therefore, we cannot distinguish the isolated effects of the LITT in our results. We had to overcome a series of challenges to apply this technology in the spine. Percutaneous techniques are commonly used in performing minimally invasive spinal procedures, but all image guidance modalities and instruments are based on CT; thus, there is no current image guidance method that allows MRI to acquire the parameters for navigation. We obtained a high-resolution CT scan and fused it with anteroposterior and lateral fluoroscopic images, allowing the use of CT to identify the contour of the spinal canal.

The length of the procedure depends on a learning curve. We found it extremely difficult to perform SLITT in patients with morbid obesity, since fitting the patient through the MRI bore is challenging and the quality of the thermal image depends on the proximity of the MRI coil to the target. We recommend limiting this technique to patients weighing less than $300 \mathrm{lbs}$. The presence of 
titanium hardware overlying the epidural tumor creates an artifact that precludes the acquisition of thermal images; therefore, utilization of this technique is somewhat limited in cases of tumor recurrence, since most cases treated with conventional surgery involve the placement of spinal hardware for stabilization. Optimizing image guidance for probe placement and the localization studies to identify the correct plane of the catheter can significantly shorten the operative time. One useful strategy is placing a titanium needle inside each access cannula, which creates an artifact that aids in localizing the correct anatomical plane of the laser fiber to perform the thermal images. To obtain reliable thermal mapping, it is imperative to interrupt respiratory motion, but we limit the time for each cycle of breath holding to no more than 2 minutes. If needed, additional cycles can be performed until satisfactory coverage of the tumor is achieved.

In general, heat delivered by the laser requires water to dissipate; ${ }^{26}$ therefore, there are differences in the size of the tumor covered by a single fiber in different organs. In general, bone is an unfavorable tissue for large coverage with this method. Our results indicate that each fiber can cover an approximately 8 - to $10-\mathrm{mm}$ radius, assuming that heat will spread in an oval shape around the fiber. Unlike in the brain, there is virtually no morbidity involved in taking multiple trajectories to achieve coverage of the epidural tumor. We have used up to 6 punctures and bilateral transpedicular approaches without any complication. Even highly vascular tumors tolerated multiple punctures well, and no significant bleeding was encountered. If bleeding occurred, we placed hemostatic foam and inserted the titanium needle inside the access cannula for a few minutes to tamponade the bleeding source.

Another interesting observation was the spinal cord's location in a completely different compartment than the epidural tumor. We observed no swelling of ablated tumor into the spinal canal, probably because these tumors are contained by the posterior longitudinal ligament, epidural veins, and periosteum, a distinction from the ablation effect in intrinsic brain tumors, in which steroids are recommended to decrease tumor swelling. Moreover, the CSF serves as a heat sink, and in the levels where it was present, we could see on thermal imaging the heat contouring the spinal canal and dissipating in the CSF. The system can be used to monitor up to 3 safety points, and once the temperature reaches the predefined limit of $50^{\circ} \mathrm{C}$, the system automatically shuts off. We theorize that heat spreads as an ellipse or a circle; therefore, its most medial extent is at the level of the axial plane of the fiber in relation to the spinal cord. We monitor our safety points along the exact axial plane of the fiber because failure to do so could result in severe neurological damage. One of our patients (Case 5) had no CSF interposed between the C-2 tumor and the spinal cord, and her tumor was located exclusively in the epidural space; therefore, there was no pseudocapsule of posterior longitudinal ligament, periosteum, and epidural veins. In this case, we set the laser to stop immediately at the interface between the tumor and the spinal cord. Unfortunately, T2 signal changes developed in the spinal cord, which were associated with tingling in the lower extremity compatible with a partial spinothalamic dysfunction.
We believe that a more conservative approach is needed in such cases, utilizing lower safety limit temperatures (that is, $45^{\circ}-46^{\circ} \mathrm{C}$ ) and placing the voxel monitoring the temperature inside the tumor and not in the interface between the tumor and the spinal cord.

The majority of our patients had symptomatic improvement of pain shortly after the procedure. We obtained VAS scores for pain and quality of life in all patients within 30 and 60 days after treatment, with most patients reporting improvement immediately after the ablation and a brief pain flare-up around 3-7 days after the SSRS. Overall, subjective patient satisfaction was very high, and the hospital stay was shorter than that for conventional surgery. The only exception occurred in the patient who did not respond to treatment and developed intractable radicular pain (Case 8 ). In this case, we performed the standard surgical treatment for which the patient would have been considered, without adverse events or significant additional delay in the initiation of chemotherapy.

All patients developed a ring-enhancing pattern on the postprocedure spinal MRI (Fig. 4). The nature of this contrast enhancement in the epidural space is uncertain and could represent engorgement of the epidural veins, inflammation related to heat damage, the development of scar in the posterior longitudinal ligament, residual viable tumor, or a combination of these factors. We have had 4 patients with 6 months of imaging follow-up, and no change on the thickness of this epidural enhancement was noted.

This report represents our initial experience with this technique, and we recognize several limitations, including the small size of the cohort, the short follow-up, the lack of randomization, no direct comparison with surgery, and no standardization in the time for postprocedure SSRS. We believe this report lays the foundation for further investigation through a randomized prospective study to compare this technique with separation surgery prior to SSRS.

\section{Conclusions}

In summary, we present a minimally invasive technique associated with low surgical morbidity, a short hospital stay, and minimal impact on quality of life in treating patients with high-grade ESCC. We believe this procedure can be an alternative to separation surgery in patients without neurological deficits prior to SSRS, especially in cases with progressive systemic disease, in which conventional surgery would pose a high risk for complications and lead to an interruption of or delays in the delivery of the intended oncological treatment.

\section{References}

1. Ahrar K, Stafford RJ: Magnetic resonance imaging-guided laser ablation of bone tumors. Tech Vasc Interv Radiol 14:177-182, 2011

2. Akeboshi M, Yamakado K, Nakatsuka A, Hataji O, Taguchi $\mathrm{O}$, Takao M, et al: Percutaneous radiofrequency ablation of lung neoplasms: initial therapeutic response. J Vasc Interv Radiol 15:463-470, 2004

3. Akeyson EW, McCutcheon IE: Single-stage posterior vertebrectomy and replacement combined with posterior instru- 
mentation for spinal metastasis. J Neurosurg 85:211-220, 1996

4. Bilsky M, Smith M: Surgical approach to epidural spinal cord compression. Hematol Oncol Clin North Am 20:13071317, 2006

5. Bilsky MH, Laufer I, Burch S: Shifting paradigms in the treatment of metastatic spine disease. Spine (Phila Pa 1976) 34 (22 Suppl):S101-S107, 2009

6. Bilsky MH, Laufer I, Fourney DR, Groff M, Schmidt MH, Varga PP, et al: Reliability analysis of the epidural spinal cord compression scale. J Neurosurg Spine 13:324-328, 2010

7. Carpentier A, Chauvet D, Reina V, Beccaria K, Leclerq D, McNichols RJ, et al: MR-guided laser-induced thermal therapy (LITT) for recurrent glioblastomas. Lasers Surg Med 44:361-368, 2012

8. Carpentier A, McNichols RJ, Stafford RJ, Itzcovitz J, Guichard JP, Reizine D, et al: Real-time magnetic resonance-guided laser thermal therapy for focal metastatic brain tumors. Neurosurgery 63:ONS21-ONS29, 2008

9. Chang EL, Shiu AS, Mendel E, Mathews LA, Mahajan A, Allen PK, et al: Phase I/II study of stereotactic body radiotherapy for spinal metastasis and its pattern of failure. J Neurosurg Spine 7:151-160, 2007

10. Curry DJ, Gowda A, McNichols RJ, Wilfong AA: MR-guided stereotactic laser ablation of epileptogenic foci in children. Epilepsy Behav 24:408-414, 2012

11. EuroQol Group: EuroQol-a new facility for the measurement of health-related quality of life. Health Policy 16:199208, 1990

12. Fisher CG, DiPaola CP, Ryken TC, Bilsky MH, Shaffrey CI, Berven SH, et al: A novel classification system for spinal instability in neoplastic disease: an evidence-based approach and expert consensus from the Spine Oncology Study Group. Spine (Phila Pa 1976) 35:E1221-E1229, 2010

13. Fourney DR, Abi-Said D, Lang FF, McCutcheon IE, Gokaslan ZL: Use of pedicle screw fixation in the management of malignant spinal disease: experience in 100 consecutive procedures. J Neurosurg 94 (1 Suppl):25-37, 2001

14. Frankel HL, Hancock DO, Hyslop G, Melzak J, Michaelis LS, Ungar GH, et al: The values of postural reduction in the initial management of closed injuries of the spine with paraplegia and tetraplegia. Paraplegia 7:179-192, 1969

15. Garg AK, Wang XS, Shiu AS, Allen P, Yang J, McAleer MF, et al: Prospective evaluation of spinal reirradiation by using stereotactic body radiation therapy: The University of Texas MD Anderson Cancer Center experience. Cancer 117:35093516, 2011

16. Gerszten PC, Burton SA, Ozhasoglu C, Vogel WJ, Welch WC, Baar J, et al: Stereotactic radiosurgery for spinal metastases from renal cell carcinoma. J Neurosurg Spine 3:288295, 2005

17. Gerszten PC, Burton SA, Ozhasoglu C, Welch WC: Radiosurgery for spinal metastases: clinical experience in 500 cases from a single institution. Spine (Phila Pa 1976) 32:193-199, 2007

18. Gerszten PC, Mendel E, Yamada Y: Radiotherapy and radiosurgery for metastatic spine disease: what are the options, indications, and outcomes? Spine (Phila Pa 1976) 34 (22 Suppl):S78-S92, 2009

19. Gilbert RW, Kim JH, Posner JB: Epidural spinal cord compression from metastatic tumor: diagnosis and treatment. Ann Neurol 3:40-51, 1978

20. Latini P, Maranzano E, Ricci S, Aristei C, Checcaglini F, Panizza BM, et al: Role of radiotherapy in metastatic spinal cord compression: preliminary results from a prospective trial. Radiother Oncol 15:227-233, 1989

21. Laufer I, Iorgulescu JB, Chapman T, Lis E, Shi W, Zhang Z, et al: Local disease control for spinal metastases following "separation surgery" and adjuvant hypofractionated or highdose single-fraction stereotactic radiosurgery: outcome analysis in 186 patients. J Neurosurg Spine 18:207-214, 2013

22. Laufer I, Rubin DG, Lis E, Cox BW, Stubblefield MD, Yamada Y, et al: The NOMS framework: approach to the treatment of spinal metastatic tumors. Oncologist 18:744-751, 2013

23. Maranzano E, Bellavita R, Rossi R: Radiotherapy alone or surgery in spinal cord compression? The choice depends on accurate patient selection. J Clin Oncol 23:8270-8274, 2005

24. Maranzano E, Latini P, Checcaglini F, Ricci S, Panizza BM, Aristei C, et al: Radiation therapy in metastatic spinal cord compression. A prospective analysis of 105 consecutive patients. Cancer 67:1311-1317, 1991

25. Maranzano E, Latini P, Perrucci E, Beneventi S, Lupattelli M, Corgna E: Short-course radiotherapy (8 Gy x 2 ) in metastatic spinal cord compression: an effective and feasible treatment. Int J Radiat Oncol Biol Phys 38:1037-1044, 1997

26. McNichols RJ, Gowda A, Kangasniemi M, Bankson JA, Price RE, Hazle JD: MR thermometry-based feedback control of laser interstitial thermal therapy at $980 \mathrm{~nm}$. Lasers Surg Med 34:48-55, 2004

27. Molina CA, Gokaslan ZL, Sciubba DM: Diagnosis and management of metastatic cervical spine tumors. Orthop Clin North Am 43:75-87, viii-ix, 2012

28. Moussazadeh N, Laufer I, Yamada Y, Bilsky MH: Separation surgery for spinal metastases: effect of spinal radiosurgery on surgical treatment goals. Cancer Contr 21:168-174, 2014

29. Nakatsuka A, Yamakado K, Takaki H, Uraki J, Makita M, Oshima F, et al: Percutaneous radiofrequency ablation of painful spinal tumors adjacent to the spinal cord with realtime monitoring of spinal canal temperature: a prospective study. Cardiovasc Intervent Radiol 32:70-75, 2009

30. Nguyen QN, Shiu AS, Rhines LD, Wang H, Allen PK, Wang $\mathrm{XS}$, et al: Management of spinal metastases from renal cell carcinoma using stereotactic body radiotherapy. Int J Radiat Oncol Biol Phys 76:1185-1192, 2010

31. Omeis I, Bekelis K, Gregory A, McGirt M, Sciubba D, Bydon A, et al: The use of expandable cages in patients undergoing multilevel corpectomies for metastatic tumors in the cervical spine. Orthopedics 33:87-92, 2010

32. Patchell RA, Tibbs PA, Regine WF, Payne R, Saris S, Kryscio RJ, et al: Direct decompressive surgical resection in the treatment of spinal cord compression caused by metastatic cancer: a randomised trial. Lancet 366:643-648, 2005

33. Rades D, Fehlauer F, Stalpers LJ, Wildfang I, Zschenker O, Schild SE, et al: A prospective evaluation of two radiotherapy schedules with 10 versus 20 fractions for the treatment of metastatic spinal cord compression: final results of a multicenter study. Cancer 101:2687-2692, 2004

34. Rades D, Freundt K, Meyners T, Bajrovic A, Basic H, Karstens JH, et al: Dose escalation for metastatic spinal cord compression in patients with relatively radioresistant tumors. Int J Radiat Oncol Biol Phys 80:1492-1497, 2011

35. Rades D, Karstens JH, Alberti W: Role of radiotherapy in the treatment of motor dysfunction due to metastatic spinal cord compression: comparison of three different fractionation schedules. Int J Radiat Oncol Biol Phys 54:1160-1164, 2002

36. Rosenthal D, Callstrom MR: Critical review and state of the art in interventional oncology: benign and metastatic disease involving bone. Radiology 262:765-780, 2012

37. Sahgal A, Bilsky M, Chang EL, Ma L, Yamada Y, Rhines $\mathrm{LD}$, et al: Stereotactic body radiotherapy for spinal metastases: current status, with a focus on its application in the postoperative patient. J Neurosurg Spine 14:151-166, 2011

38. Vogl TJ, Dommermuth A, Heinle B, Nour-Eldin NE, Lehnert T, Eichler K, et al: Colorectal cancer liver metastases: longterm survival and progression-free survival after thermal ablation using magnetic resonance-guided laser-induced in- 
terstitial thermotherapy in 594 patients: analysis of prognostic factors. Invest Radiol 49:48-56, 2014

39. Wang XS, Rhines LD, Shiu AS, Yang JN, Selek U, Gning I, et al: Stereotactic body radiation therapy for management of spinal metastases in patients without spinal cord compression: a phase 1-2 trial. Lancet Oncol 13:395-402, 2012

40. Yamada Y, Bilsky MH, Lovelock DM, Venkatraman ES, Toner S, Johnson J, et al: High-dose, single-fraction imageguided intensity-modulated radiotherapy for metastatic spinal lesions. Int J Radiat Oncol Biol Phys 71:484-490, 2008

41. Yamakado K, Nakatsuka A, Akeboshi M, Takeda K: Percutaneous radiofrequency ablation of liver neoplasms adjacent to the gastrointestinal tract after balloon catheter interposition. J Vasc Interv Radiol 14:1183-1186, 2003

42. Young RF, Post EM, King GA: Treatment of spinal epidural metastases. Randomized prospective comparison of laminectomy and radiotherapy. J Neurosurg 53:741-748, 1980

\section{Author Contributions}

Conception and design: Tatsui, Rhines. Acquisition of data: Tatsui, Stafford, Li, Amini, Cowles. Analysis and interpretation of data: Tatsui, Stafford, Li, Amini. Drafting the article: Tatsui, Sellin. Critically revising the article: Tatsui, Stafford, Li, Amini, Rao, Ghia, Brown, Lee, Weinberg, Rhines. Reviewed submitted version of manuscript: Tatsui, Sellin, Amini, Rao, Suki, Ghia, Brown, Lee, Weinberg, Rhines. Approved the final version of the manuscript on behalf of all authors: Tatsui. Statistical analysis: Suki, Lee.

\section{Correspondence}

Claudio E. Tatsui, Department of Neurosurgery, The University of Texas M.D. Anderson Cancer Center, 1515 Holcombe Blvd., Unit 442, Houston, TX 77030. email: cetatsui@mdanderson.org. 